\title{
Size effects investigated by Raman spectroscopy in Bi nanocrystals
}

\author{
E. Haro-Poniatowski, * M. Jouanne, J. F. Morhange, and M. Kanehisa \\ Laboratoire des Milieux Désordonnés et Hetérogènes, Unité Mixte UPMC-CNRS, UMR 7603, Université Pierre et Marie Curie, \\ 4 Place Jussieu, 75252 Paris Cédex 05, France \\ R. Serna and C. N. Afonso \\ Instituto de Optica, CSIC, Serrano 121, 28006 Madrid, Spain
}

(Received 9 December 1998; revised manuscript received 22 April 1999)

\begin{abstract}
Bismuth nanocrystals, embedded in amorphous germanium thin films and synthesized by pulsed-laser deposition, are investigated by Raman spectroscopy. Raman spectra are strongly dependent upon the size of the deposited nanocrystals. For the larger nanocrystals, two peaks corresponding to the optical phonons $A_{1 g}$ and $E_{g}$ can be observed. As the size of the particles decreases, the $E_{g}$ mode shifts to higher frequencies while the $A_{1 g}$ mode shifts to lower frequencies. Such behavior is a result of the curvature of the respective phonon-dispersion curves in $\mathrm{Bi}$. A third size-dependent low-frequency mode has also been observed and attributed to acoustic phonons confined in Bi nanocrystals. [S0163-1829(99)13933-X]
\end{abstract}

\section{INTRODUCTION}

Nanocrystals (NC's) are currently attracting much interest because of their specific properties, resulting from the quantum confinement. As typical examples, silicon NC's show light emission in the visible range, ${ }^{1}$ metallic and semiconductor NC's embedded in dielectrics exhibit nonlinear optical properties. ${ }^{2-4} \mathrm{NC}$ 's are also used as catalytic supports because of their large surface to volume ratio. ${ }^{5}$ It has been shown that the properties of these materials depend strongly on the film thickness and on the deposition technique that ultimately controls the final structure (grain size). Such structures can be prepared in various ways like ion implantation, ${ }^{6}$ sputtering, ${ }^{7}$ sol-gel processes, ${ }^{8}$ and pulsed-laser deposition (PLD). ${ }^{4,9,10}$ This last technique has remarkable advantages for the preparation of materials with complex structures ${ }^{11}$ and it is specially suited for the deposition of films for optical applications. ${ }^{12}$

Structural characterization of nanocomposite films can be performed using various techniques including $\mathrm{x}$ rays, highresolution transmission electronic microscopy (HRTEM), and Raman spectroscopy. This last technique is well suited to the study of nanometric-sized systems that show characteristic properties derived from the three-dimensional confinement of electrons and holes as well as of phonons. Raman scattering from confined acoustic phonons has been already reported for some metal ${ }^{2}$ and semiconductor NC's. ${ }^{13,14}$ In the present work, PLD has been used for the synthesis of nanocomposite films with $\mathrm{Bi}$ in the form of NC's embedded in amorphous Ge. 10,15 $^{\text {. }}$

$\mathrm{Bi}$ in thin-film configuration has been intensively studied mainly because of its special thermoelectrical properties. Superconductivity and quantum-size phenomena have been also reported. ${ }^{16,17}$ Good quality Bi films have been recently prepared by PLD and, in contrast to films prepared by other techniques, their optical properties were shown to be independent of their thickness. ${ }^{18}$

The aim of this paper is to investigate the sensitivity of the Raman-scattering response to the size of Bi (NC's) with average sizes ranging from 2.3 to $23 \mathrm{~nm}$. For this study, the Bi NC's have been embedded in an amorphous Ge matrix, this solid-state composite system has the advantage that it is simple, has been very well characterized, ${ }^{10,15,19}$ and therefore allows the study of the size effects on both optical and acoustic phonons. The evolution of the optical modes of the crystalline phase was analyzed, and an attempt to relate the observed low-frequency band with the theory of the normal modes of a vibrating sphere is reported.

\section{EXPERIMENTS}

The specimens are thin nanocomposite films prepared by alternate PLD. An ArF excimer laser [193 nm, 12-ns fullwidth half maximum (FWHM), $5-\mathrm{Hz}$ repetition rate] was used with an average energy density of $2 \mathrm{~J} \mathrm{~cm}^{-2}$. The deposition was performed in vacuum $\left(7 \times 10^{-7}\right.$ Torr $)$ onto $\mathrm{Si}(100)$ substrates held at room temperature. The substratetarget distance was set to $32 \mathrm{~mm}$. High-purity targets of Ge and $\mathrm{Bi}$ were mounted in a computer-controlled multiple holder that allowed us to alternately expose each target to the laser beam. In order to increase the amount of Bi NC's and therefore the Raman signal related to them, a layered structure was designed in which a layer of Bi NC's was deposited and subsequently covered by a Ge continuous layer, this structure being repeated up to five times. The last deposited layer was always $\mathrm{Ge}$ in order to ensure that the Bi NC's are covered with the matrix element, thus preventing oxidation. The deposition sequence involved a number of pulses on the Bi target, ranging from 12 to 200 pulses, followed by a fixed number of pulses on the Ge target (100 pulses). Figure 1 shows a plan view high-resolution transmission electron microscopy image of the film deposited using 50 pulses on the $\mathrm{Bi}$ target. The image shows a pale random contrast in the background corresponding to the amorphous Ge together with darker contrast regions in which crystal lattice fringes are visible. These regions correspond to fully ordered $\mathrm{Bi}$ NC's. As the number of pulses on the Bi target increases, the size of the obtained NC's grows. Regarding the NC's shape, 


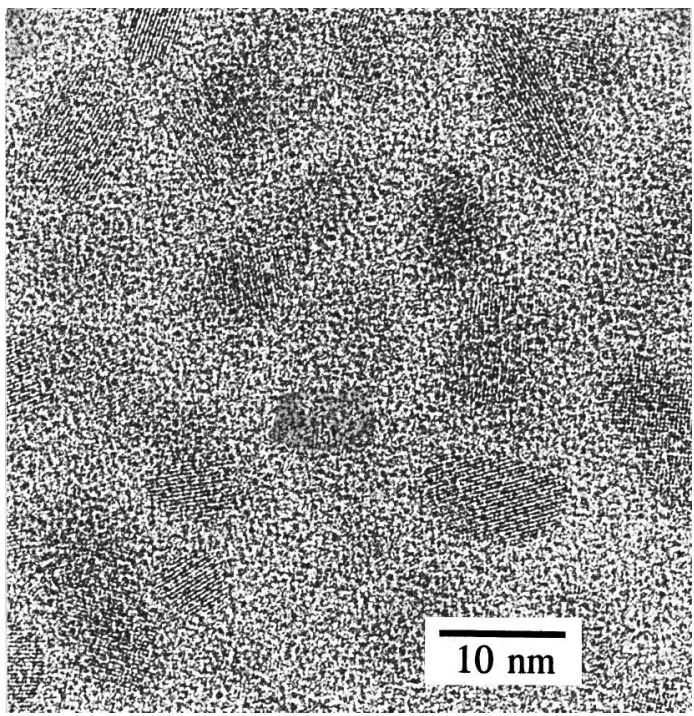

FIG. 1. Plan view of high-resolution microscopy bright field image of a nanocomposite film deposited using 50 pulses on the $\mathrm{Bi}$ target per layer.

it was found that the Bi NC's are oblate ellipsoids with two similar axes in the film plane and a shorter axis in the film normal direction. ${ }^{10}$ The total thickness of the samples ranged from $20 \mathrm{~nm}$ (BG12) to $44 \mathrm{~nm}$ (BG200). Table I includes a summary of the studied films, their deposition conditions, and the NC's mean diameter as reported elsewhere, ${ }^{10}$ as well as the mean height of the NC (shorter axis of the ellipsoid), as determined by Rutherford backscattering (RBS). X-raydiffraction measurements on those same samples showed that the NC's are partially ordered, having all the $\left\langle\begin{array}{llll}1 & 1 & 0\end{array}\right)$ axes of the rhombohedric structure (corresponding to the $\left\langle\begin{array}{lll}1 & 0 & 2\end{array}\right\rangle$ axis of the hexagonal equivalent structure) normal to the plane of deposition.

The absorption coefficient, as measured by spectroscopic ellipsometry, ${ }^{19}$ gives a penetration depth at the Raman laser wavelength of approximately $20 \mathrm{~nm}$ for the sample with the lowest number of pulses on the Bi target (BG12) and $13 \mathrm{~nm}$ for the sample with the largest number of pulses on the $\mathrm{Bi}$ target (BG200). This means that no signal from the Si substrate is essentially recorded for the thickest films.

TABLE I. Summary of the nanocomposite films. The second column shows the number of pulses on the Bi target used to deposit the NC's. The number of pulses on the Ge target was constant (100 pulses) and the films have a total of five alternate layers of Bi NC's. The third and fourth columns contain the Bi NC's mean diameter measured from the HRTEM images (taken from Ref. 10) and their mean height, measured by RBS.

\begin{tabular}{lccc}
\hline \hline Sample & $\begin{array}{c}\text { No. of pulses } \\
\text { on Bi } \\
\text { target per layer }\end{array}$ & $\begin{array}{c}\text { Mean height } \\
\text { of Bi } \\
\text { NC's (nm) }\end{array}$ & $\begin{array}{c}\text { Mean diameter } \\
\text { of Bi } \\
\text { NC's (nm) }\end{array}$ \\
\hline BG12 & 12 & 0.50 & $2.3 \pm 0.8$ \\
BG25 & 25 & 0.84 & $2.5 \pm 0.8$ \\
BG50 & 50 & 1.73 & $7 \pm 2$ \\
BG100 & 100 & 3.73 & $17 \pm 2$ \\
BG200 & 200 & 6.18 & $23 \pm 2$ \\
\hline
\end{tabular}

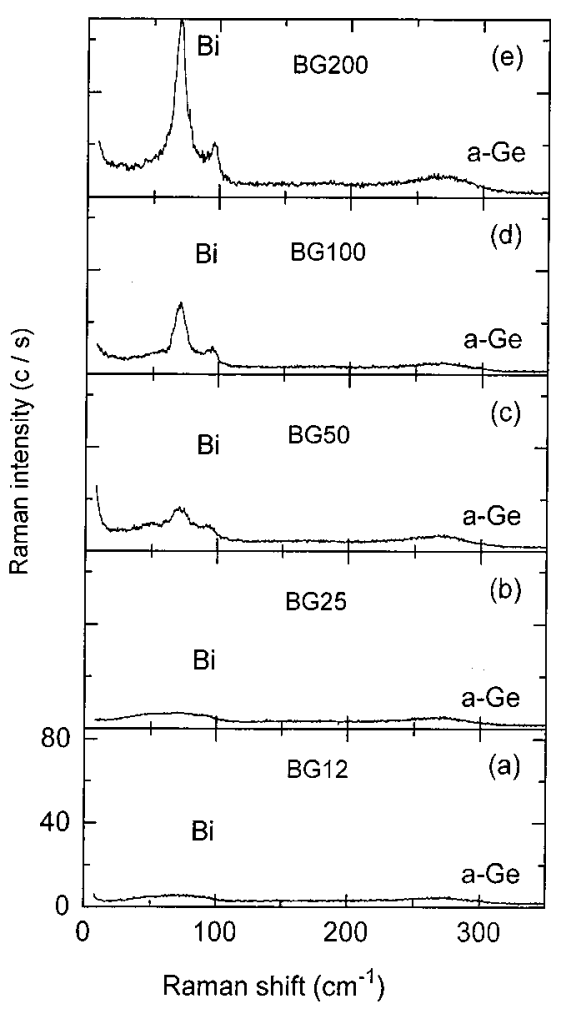

FIG. 2. Raman spectra of the nanocomposite films as a function of the mean height of the Bi NC's. (a) $0.50 \mathrm{~nm}$, (b) $0.84 \mathrm{~nm}$, (c) $1.73 \mathrm{~nm}$, (d) $3.73 \mathrm{~nm}$, (e) $6.18 \mathrm{~nm}$.

Raman spectroscopy measurements were performed in vacuum at room temperature using a Jobin-Yvon U1000 double monochromator. The 514.5-nm line of an argon (Spectra Physics) laser was used as the excitation source at a power level of $100 \mathrm{~mW}$. The size of the laser spot at the surface of the sample was of the order of $100 \mu \mathrm{m}$. Due to the low Raman signal level (weaker signals correspond to scattering by a few atomic layers), each spectrum was the result of the addition of several scans. Some measurements were performed at low temperature, around $20 \mathrm{~K}$. For comparison purposes, the Raman spectrum of a Bi-single-crystal sample was also recorded.

\section{RESULTS}

In Fig. 2, the raw Raman spectra of the samples are presented as a function of the Bi NC size: (a) $2.3 \mathrm{~nm}$, (b) 2.5 $\mathrm{nm}$, (c) $7 \mathrm{~nm}$, (d) $17 \mathrm{~nm}$, and (e) $23 \mathrm{~nm}$. In this figure, for comparison purposes, all spectra are shown at the same Raman intensity scale $(0-80$ counts/s). In Fig. 2(a), two very low-intensity broadbands are present. The first, centered at $270 \mathrm{~cm}^{-1}$, corresponds to the optical part of the Raman spectrum of amorphous germanium (high-frequency region) while the second, around $70 \mathrm{~cm}^{-1}$, corresponds to the acoustical part of the same spectrum as well as to the Bi spectrum. Considering the small intensity of the high-frequency part of the $a$-Ge spectrum, and that the intensity of the lowfrequency part of the $a$-Ge spectrum is at least two times smaller, ${ }^{20}$ these latter contributions can be neglected and the band around $70 \mathrm{~cm}^{-1}$ can be fully attributed to the Bi NC's.

As the Bi NC size grows, the intensity of the correspond- 


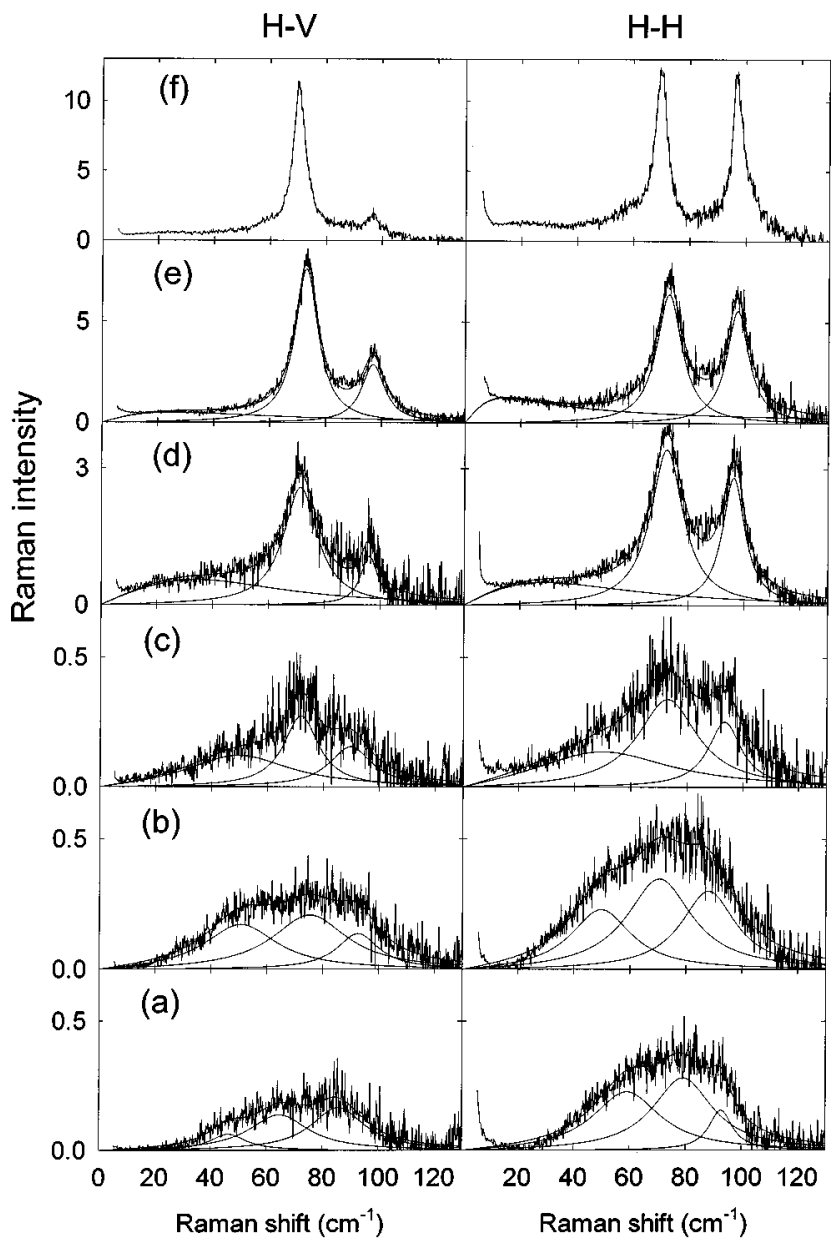

FIG. 3. Low-frequency Raman spectra of the films in parallel and perpendicular polarizations, as a function of the mean height of the Bi NC's: $0.50 \mathrm{~nm}$ (a), $0.84 \mathrm{~nm}$ (b), $1.73 \mathrm{~nm}$ (c), $3.73 \mathrm{~nm}$ (d), $6.18 \mathrm{~nm}(\mathrm{e})$, and Bi single crystal (f).

ing band (around $70 \mathrm{~cm}^{-1}$ ) slightly increases, as can be seen in Fig. 2(b). In Fig. 2(c) one clearly observes that the Bi band splits into three subbands pointing at 50,70 , and $95 \mathrm{~cm}^{-1}$, approximately. The bands pointing at 70 and $95 \mathrm{~cm}^{-1}$ can be unambiguously attributed to the $E_{g}$ and $A_{1 g}$ modes of the $\mathrm{Bi}$ material. ${ }^{21-23}$ The lowest-frequency band near $50 \mathrm{~cm}^{-1}$ has no obvious origin. As the size of the Bi NC's increases further, the two highest frequency bands evolve into narrow and intense peaks [Figs. 2(d) and 2(e)]. The low-frequency band shifts towards low frequency. In contrast, the $a$-Ge band centered at $270 \mathrm{~cm}^{-1}$ has roughly remained constant in intensity, shape, and position.

In order to analyze in detail the Bi NC's response, Fig. 3 shows the low-frequency Raman spectra $\left(0-120 \mathrm{~cm}^{-1}\right)$ of the five samples together with the spectrum of a $\mathrm{Bi}$ single crystal recorded both in parallel $(H-V)$ and perpendicular polarizations $(H-H)$. The Raman spectra were reduced by the Bose-Einstein factor in order to get the true profile lines. In this figure, it is clearly seen that the optical modes of the crystalline $\mathrm{Bi}$ are not perfectly resolved for the films made of Bi NC's of small size [Figs. 3(a) and 3(b)]. One broad peak with a weak polarization dependence is the only observed feature. As the NC size is increased [Figs. 3(c) and 3(d)], the peaks corresponding to the optical modes of Bi become apparent and a similar polarization dependence to that of the $\mathrm{Bi}$ single crystal [Fig. 3(a)] is observed. On the Bi single crystal, the two main peaks observed are the well-known modes $E_{g}\left(70 \mathrm{~cm}^{-1}\right)$ and $A_{1 g}\left(97 \mathrm{~cm}^{-1}\right)$ of the $\mathrm{Bi}$, as previously described. Additionally, a continuum of broadbands centered around 22 and $67 \mathrm{~cm}^{-1}$ extending over more than $100 \mathrm{~cm}^{-1}$ is also observed. Various combinations of phonons, e.g., $A_{1 g}(\Gamma)-E_{g}(\Gamma)$ at $27 \mathrm{~cm}^{-1}, 2 \mathrm{TA}(X)$ at $45 \mathrm{~cm}^{-1}$, and $2 \mathrm{LA}(X)$ at $67 \mathrm{~cm}^{-1}$ could be responsible for these continuum bands. ${ }^{24-26}$ To study the origin of the lowestfrequency broadband in $\mathrm{Bi}$, some Raman spectra were recorded at low temperature. The disparition of these bands at low temperature in the case of the Bi single crystal confirms a possible second-order Raman origin. On the contrary, for the thin nanocomposite films, a low-frequency band is still present at low temperature. This low-temperature behavior thus reveals a different origin for the lowest-frequency band in monocrystalline and nanocrystalline bismuth.

\section{DISCUSSION}

To understand the features of the Raman spectra shown in Fig. 3, we have fitted the observed reduced spectra to a sum of phonon spectral functions, i.e., the imaginary part of the retarded Green's function: ${ }^{27}$

$$
\begin{aligned}
& -\sum_{i} A_{i} \operatorname{Im}\left[\frac{1}{2 \omega_{0 i}}\left(\frac{1}{\omega-\omega_{0 i}+i \Gamma_{i}}-\frac{1}{\omega+\omega_{0 i}+i \Gamma_{i}}\right)\right] \\
& =-\sum_{i} A_{i} \operatorname{Im}\left[\frac{1}{\left(\omega+i \Gamma_{i}\right)^{2}-\omega_{0 i}^{2}}\right] \\
& =\sum_{i} \frac{2 A_{i} \omega_{0 i} \Gamma_{i}}{\left(\omega^{2}-\omega_{0 i}^{2}-\Gamma_{i}^{2}\right)^{2}+4 \omega^{2} \Gamma_{i}^{2}} .
\end{aligned}
$$

Here $A_{i}$ is the oscillator strength of mode $i, \omega_{0 i}$, is the mode frequency, and $\Gamma_{i}$ is the width. This formula coincides with the usual damped oscillator response function ${ }^{28}$ if one puts $\omega_{0 i}^{2}+\Gamma_{i}^{2} \rightarrow \omega_{0}^{2}, 2 \Gamma_{i} \rightarrow \Gamma$. Note that Eq. (1) is not perfectly symmetric around $\omega_{0 i}$ and that $\omega_{0 i}$ is not the frequency of the maximum, especially when it is small and $\Gamma_{i}$ is large. In our case, three functions of this shape have been introduced $(i=1,2,3)$ to describe the spectra. Two of them correspond to the optical modes of $\mathrm{Bi}\left(E_{g}\right.$ and $\left.A_{1 g}\right)$. The remaining one describes the broad low-frequency band. The results of this fitting are shown in Fig. 3. For all the samples, the peak frequencies $\omega_{0 i}$ obtained from the fit, as well as the widths $\Gamma_{i}$, are summarized in Table II. The three peak frequencies have also been plotted in Fig. 4 as a function of the inverse reduced height of the $\mathrm{Bi} \mathrm{NC}$ [i.e., $2 d_{110} / h$, where $d_{110}$ $=0.328 \mathrm{~nm}$ is the distance between two adjacent (110) planes].

The major contribution in all the spectra comes from the optical modes $E_{g}$ and $A_{1 g}$. As can be seen in Fig. 4, the frequency of the $A_{1 g}$ mode shifts towards lower frequencies when the size of the NC's decreases, while the frequency of the $E_{g}$ mode $\left(\omega_{0}\right)$ slightly shifts towards higher frequencies near the zone center. This behavior observed on the $E_{g}$ mode is not frequent, the reverse effect being usually reported (decreasing of frequency as the size reduces). ${ }^{29-31}$ This specific 
TABLE II. Parameters obtained for the least-squares best fits between the Raman spectra of NC bismuth and a composition of three response functions.

\begin{tabular}{lcccccc}
\hline \hline Samples & $\omega_{1}\left(\mathrm{~cm}^{-1}\right)$ & $\Gamma_{1}\left(\mathrm{~cm}^{-1}\right)$ & $\omega_{2}\left(\mathrm{~cm}^{-1}\right)$ & $\Gamma_{2}\left(\mathrm{~cm}^{-1}\right)$ & $\omega_{3}\left(\mathrm{~cm}^{-1}\right)$ & $\Gamma_{3}\left(\mathrm{~cm}^{-1}\right)$ \\
\hline BG12 & 66.2 & 14.6 & 86.8 & 12.3 & 3.1 & 43.2 \\
BG25 & 68.5 & 18.7 & 88.5 & 17.1 & 17.0 & 52.0 \\
BG50 & 72.4 & 10.3 & 91.6 & 8.3 & 49.6 & 27.8 \\
BG100 & 71.8 & 7.2 & 96.1 & 4.5 & 48.0 & 13.3 \\
BG200 & 73.0 & 5.4 & 97.2 & 5.3 & 48.0 & 8.5 \\
\hline \hline
\end{tabular}

behavior, as well as the usually reported one, can be explained in the frame of the theory of Richter, Wong, and Ley on microcrystallites. ${ }^{30}$

In Fig. 5, the phonon dispersion curves for the monocrystalline bismuth along the $X$ direction of the Brillouin zone are represented. ${ }^{24-26}$ This direction corresponds to the direction of propagation of the Raman observed vibrations in the actual backscattering geometry along the $\langle 110\rangle$ axes of the nanocrystals. The phonon dispersion curve of the $E_{g}$ mode of the $\mathrm{Bi}$ has a curvature towards high frequencies near the $\Gamma$ point. $^{24-26}$ The other mode $A_{1 g}$ has a tendency to shift towards lower frequencies reflecting the more common behavior of the dispersion branch corresponding to this mode. The form of the frequency variations for the $E_{g}$ and $A_{g 1}$ peaks that reflects the curvature of the optical phonon dispersion curves was already reported on ultrathin layers of $\mathrm{Bi}$ deposited on carbon by dc sputtering. ${ }^{21,22}$

The $\mathrm{Bi}$ embedded in our nanocomposite films is in the crystalline state as has been shown by the observation of reticular planes by HRTEM (Fig. 1 and Ref. 10). This is in agreement with the present Raman results that show that the optical phonon peaks in Fig. 3 comply with the Raman selection rules regarding the two different polarizations, even for mean height as low as $1.7 \mathrm{~nm}$ [Fig. 3(c)]. This last ob-

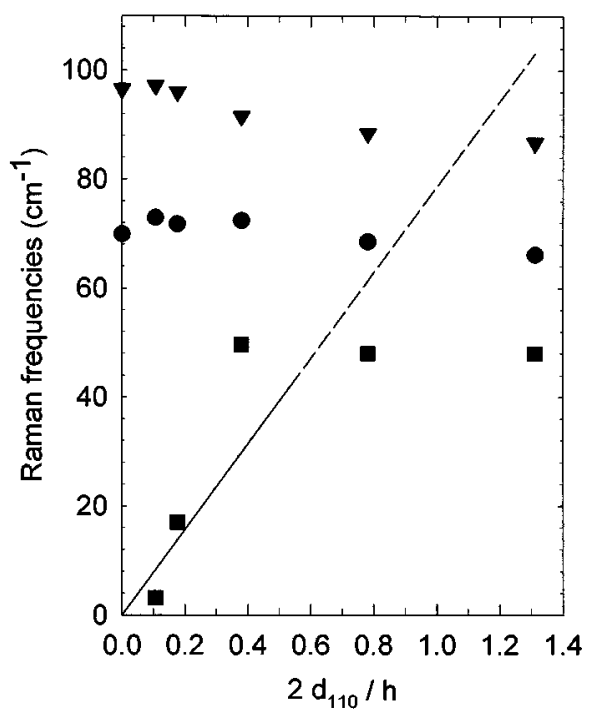

FIG. 4. Raman frequencies dependence of the $A_{1 g}$ (triangles), $E_{g}$ (circles) optical modes, and the low-frequency mode (squares) as a function of the reduced height of the NO. The continuous and dashed straight line represents the theoretical dependence of the $j$ $=0$ spheroidal vibration of a clamped sphere. servation is in contrast with earlier reports ${ }^{21,22}$ that were studied on dc-sputtered Bi ultrathin films with "equivalent thickness" from 0.2 to $1.3 \mathrm{~nm}$. For those films, Bi-cluster formation was evidenced by $\mathrm{x}$-ray photoemission and TEM. Raman spectra of films of equivalent thickness below 0.8 $\mathrm{nm}$, corresponding to clusters of sizes in the range of 5.5-6.0 $\mathrm{nm}$ showed a broadband similar to the one belonging to an amorphous structure. From the analysis of the spectrum shape, intensity, and polarization dependence it was concluded that, in those ultrathin films with clusters smaller than 5.5-6.0 $\mathrm{nm}$, there is a transition from nanocrystalline to amorphouslike clusters. ${ }^{21}$ Such a transition does not seem to occur in our Bi NC's. This fact can be due to the difference in the preparation methods that can lead to a different kinetics of nucleation and growth. It was recently reported that $\mathrm{Bi}$ films of same thickness prepared either by dc sputtering or by PLD have a different morphology. The films grown by PLD have a smooth surface state and show micron-sized laminar grains while dc-sputtered films are rougher and show nanometer-sized columnar grains. ${ }^{18}$

As mentioned before, the low-frequency band recorded on NC's Bi has a different behavior than for the single crystal since it shifts with the size (Fig. 3) and it does not disappear at low temperature. For the single-crystal bismuth, as discussed earlier, we attribute this band to various phonon combinations. ${ }^{24-26}$ For the nanocomposite thin films, another interpretation is needed. Considering the similarity between

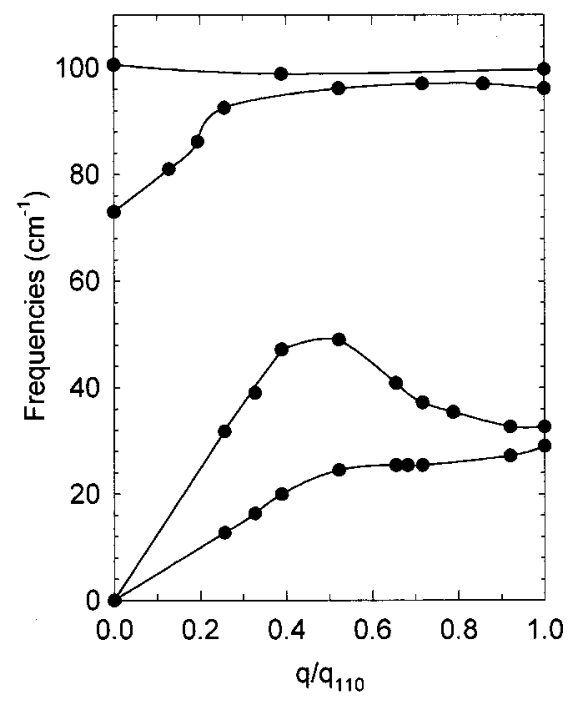

FIG. 5. Phonon dispersion curves of Bi along the (110) direction obtained by neutron-scattering experiments taken from Refs. 25 and 26. The continuous lines are only a guide for the eyes. 
the LA acoustical dispersion branch and the frequency variation of this mode with the NC size, we can attribute this band to the vibrational modes of small-sized NC's. ${ }^{3,32}$ Such modes can also be regarded as confined acoustic modes. The intensity of this mode decreases when the NC size increases as the confinement becomes less effective. In order to explore this possibility, we have calculated the highest frequency $\left(\sigma_{s}^{0}\right)$ of the two fundamental spheroidal Raman-active modes of a clamped sphere of $\mathrm{Bi}$, using the classical theory of Lamb. ${ }^{33}$ The clamping of the sphere assumes that the $a$-Ge matrix is much more rigid than the Bi NC's. For this calculation, the sound velocities were extrapolated from the linear part of the acoustical branches of the dispersion curves ${ }^{24,25}$ to $\mathrm{V}_{\mathrm{LA}}$ $=2090 \mathrm{~m} / \mathrm{s}$ and $\mathrm{v}_{\mathrm{TA}}=1360 \mathrm{~m} / \mathrm{s}$, which agree well with the values of $\mathrm{v}_{1}=2180 \mathrm{~m} / \mathrm{s}$ and $\mathrm{v}_{t}=1100 \mathrm{~m} / \mathrm{s}$ given for polycrystalline bismuth in Ref. 34 . The results of the calculation gives for the spheroidal mode,

$$
\sigma_{0}^{s}=25.8\left(\frac{1}{R}\right)
$$

where $R$ is in nanometers and $\sigma_{0}^{s}$ in $\mathrm{cm}^{-1}$. Assuming that the oblate ellipsoids of short axis $h$ can be assimilated as spheres of radius $R=h / 2$, we have plotted Eq. (2) on Fig. 4. Taking into account that only the confinement along the shortest axis has been considered and the fact that there are no adjustable parameters, we can consider the agreement between theory and experiment good. The large asymmetry of the ellipsoids does not allow us to perform the quadrupolar correction ${ }^{35}$ that would otherwise improve the model.

When the height $h$ becomes smaller, experimental points saturate and deviate from Eq. (2). This is understood from Fig. 5. For smaller $h$, only elastic waves of small wavelength (larger $q$ ) can be contained in the NC's. Now for these larger values of $q$, the dispersion curve of the acoustic branches flattens and thus the sound velocity becomes smaller. This reduces the mode frequencies.

Finally, we remark the similarity between the dispersion curves in Fig. 5 and the variation of the frequencies of the modes in Fig. 4. This is particularly the case with the LA acoustical branch in Fig. 5 and the low-frequency mode in Fig. 4. This may not be a coincidence. In fact, in superlattices, it is known that confined mode frequency for a layer of thickness $h$ is approximated by the bulk phonon frequency of wave vector $k=\pi / h$ (Ref. 36) and one can even reconstruct the phonon dispersion curve from the superlattice mode measurements. ${ }^{37}$ In our case, since the NC's have a rather flat shape, they may be roughly considered to be thin layers and the above arguments apply.

\section{CONCLUSION}

The Raman scattering of Bi nanocrystals embedded in $a-\mathrm{Ge}$ shows a strong dependence on their size. The frequency of the optical phonons shifts to lower $\left(A_{1 g}\right)$ or higher frequencies $\left(E_{g}\right)$, as the nanocrystal size decreases, depending on the curvature of their respective dispersion branches. The theoretically computed frequencies of the confined acoustic modes of a sphere agrees well with those measured on the NC in spite of the crudeness of the model.

\section{ACKNOWLEDGMENTS}

The present work has been partially supported by the Consejo Nacional de Ciencia y Tecnología of México and the Consejo Superior de Investigaciones Cientificas of Spain through the agreement CONACYT-CSIC for travel support. The authors would like to express their gratitude to M. Lemal for the x-ray-diffraction experiments.
*Permanent address: Departamento de Física, Universidad Autonoma Metropolitana Iztapalapa, Apdo. Postal 55-534, México 09340, D.F., Mexico.

${ }^{1}$ T. Shimizu-Iwayama, Y. Terao, A. Kamiya, M. Takeda, S. Nakao, and K. Saitoh, Nanostruct. Mater. 5, 307 (1995).

${ }^{2}$ M. Fujii, T. Nagareda, S. Hayashi, and K. Yamamoto, Phys. Rev. B 44, 6243 (1991)

${ }^{3}$ B. Champagnon, B. Andrianasolo, and E. Duval, Mater. Sci. Eng., B 9, 417 (1991).

${ }^{4}$ J. M. Ballesteros, R. Serna, J. Solis, C. N. Afonso, A. K. PetfordLong, D. H. Osborne, and R. F. Haglund, Jr., Appl. Phys. Lett. 71, 2445 (1997).

${ }^{5}$ P. D. L. Mercera, J. G. Van Ommen, E. B. M. Doesburg, A. J. Burggraaf, and J. R. H. Ross, Appl. Catal. 57, 127 (1990).

${ }^{6}$ Z. Pan, S. H. Morgan, D. O. Henderson, S. Y. Park, R. A. Weeks, R. H. Magruder III, and R. A. Zuhr, Opt. Mater. 4, 675 (1995).

${ }^{7}$ H. B. Liao, R. F. Xiao, J. S. Fu, P. Yu, G. K. L. Wong, and P. Sheng, Appl. Phys. Lett. 70, 1 (1997).

${ }^{8}$ G. De, L. Tapfer, M. Catalano, G. Battaglin, F. Caccavale, F. Gonella, P. Mazoldi, and R. F. Haglund, Jr., Appl. Phys. Lett. 68, 3820 (1966).

${ }^{9}$ S. Ohtsuka, K. Tsunetomo, T. Koyama, and S. Tanaka, Opt. Mater. 2, 209 (1993).

${ }^{10}$ R. Serna, T. Missana, C. N. Afonso, J. M. Ballesteros, A. K. PetfordLong, and R. C. Doole, Appl. Phys. A: Mater. Sci. Process. 66A, 43 (1998).
${ }^{11}$ D. B. Chrisey and G. K. Hubler, Pulsed Laser Deposition of Thin Films (Wiley, New York, 1994).

${ }^{12}$ C. N. Afonso, in Insulating Materials for Optoelectronics, edited by F. Agulló-López (World Scientific, Singapore, 1995), Chap. 1, pp. 1-28.

${ }^{13}$ M. Fujii, Y. Kanzawa, S. Hayashi, and K. Yamamoto, Phys. Rev. B 54, R8373 (1996).

${ }^{14}$ B. Champagnon, B. Adrianasolo, A. Ramos, M. Gandais, M. Allais, and J. P. Benoit, J. Appl. Phys. 73, 2775 (1993).

${ }^{15}$ C. N. Afonso, R. Serna, J. M. Ballesteros, and J. Solís, Proc. SPIE 3404, 74 (1998).

${ }^{16}$ Y. Liu, K. A. Megreer, B. Nease, D. B. Haviland, G. Martinez, J. W. Halley, and A. M. Geldman, Phys. Rev. Lett. 67, 2068 (1991).

${ }^{17}$ N. Garcia, Y. K. Kao, and M. Strongin, Phys. Rev. B 5, 2029 (1988).

${ }^{18}$ J. C. de Sande, T. Missana, and C. N. Afonso, J. Appl. Phys. 80, 7023 (1996).

${ }^{19}$ R. Serna, J. C. G. de Sande, J. M. Ballesteros, and C. N. Afonso, J. Appl. Phys. 84, 4509 (1998).

${ }^{20}$ J. E. Smith, Jr., M. H. Brodsky, B. L. Crowder, and M. I. Nathan, in Proceedings of the Second International Conference on Light Scattering in Solids, edited by M. Balkanski (Flammarion, Paris, 1971), p. 330.

${ }^{21}$ M. G. Mitch, S. J. Chase, J. Fortner, R. Q. Yu, and J. S. Lannin, 
Phys. Rev. Lett. 67, 875 (1991).

${ }^{22}$ J. S. Lannin, J. Non-Cryst. Solids 141, 233 (1992).

${ }^{23}$ J. S. Lannin, J. M. Calleja, and M. Cardona, Phys. Rev. B 12, 585 (1975).

${ }^{24}$ R. E. Macfarlane, in The Physics of Semimetal and Narrow Gap Semiconductors, edited by D. L. Carter and R. T. Bate (Pergamon, New York, 1971), p. 28.

${ }^{25}$ J. Sosnowski, S. Bednarski, and A. Czachor, Neutron Inelastic Scattering (IAEA, Vienna, 1958), Vol. 1, p. 157.

${ }^{26}$ A. Czachor, A. Rajca, J. Sosnowski, and A. Pindor, Acta Phys. Pol. A 43, 37 (1973).

${ }^{27}$ See, for example, A. A. Abrikosov, L. P. Gor'kov, and I. Y. Dzyaloshinskii, Quantum Field Theoretical Methods in Statistical Physics (Pergamon, Oxford, 1965).

${ }^{28}$ A. S. Barker, Jr. and R. Loudon, Rev. Mod. Phys. 41, 18 (1972).

${ }^{29}$ G. Kanellis, J. F. Morhange, and M. Balkanski, Phys. Rev. B 2 ,
1543 (1980).

${ }^{30}$ H. Richter, Z. P. Wong, and L. Ley, Solid State Commun. 39, 625 (1981).

${ }^{31}$ G. Kanellis, J. F. Morhange, and M. Balkanski, Phys. Rev. B 28, 3406 (1983).

${ }^{32}$ E. Duval, Phys. Rev. B 46, 5795 (1992).

${ }^{33}$ H. Lamb, Proc. London Math. Soc. 13, 189 (1882).

${ }^{34}$ The Handbook of Physical Quantities (CRG, Boca Raton, 1977).

${ }^{35}$ G. Mariotto, M. Montagna, G. Viliani, E. Duval, S. Lefrant, E. Rzepka, and C. Maï, Europhys. Lett. 6, 239 (1988).

${ }^{36}$ A. K. Sood, J. Menendez, M. Cardona, and K. Ploog, Phys. Rev. Lett. 54, 2111 (1985).

${ }^{37}$ See, for a review, B. Jusserand and M. Cardona, in Light Scattering in Solids V. Superlattices and Other Microstructures, edited by M. Cardona and G. Güntherodt (Springer, Berlin, 1988), p. 49 , and references cited therein. 